Revista Latinoamericana de la Papa 21 (1): 1 -14

ISSN: $1853-4961$

http://www.papaslatinas.org/revista.html

\title{
Estrategias ecológicas para el manejo del tizón tardío de la papa [Phytophthora infestans (Mont.) De Bary]
}

\author{
S. Aliaga ${ }^{1}$, F. Terrazas ${ }^{2}$, N. Ortuño ${ }^{3 / *}$
}

Recibido: 05/08/2016

Aceptado: 16/04/2017

Accesible en línea: Junio 2017

\section{Resumen}

La presente investigación se realizó con el objetivo de determinar la eficiencia de dos estrategias de control ecológico del Tizón, en la producción de papa (Solanum tuberosum subsp. andigena var. Huaycha), en las localidades de Huancarani, Link'u y Machaca del Municipio de Independencia-Ayopaya-Cochabamba. Para eso se realizó la preparación del suelo, trazado de parcelas, siembra, fertilización, labores culturales (riego, control de malezas, aporque, control fitosanitario donde aplicó los tratamientos) y la cosecha para la posterior evaluación. Se evaluaron tres tratamientos (testigo T0, estrategia orgánica T1 y estrategia combinada T2), en un diseño de bloques completos al azar con tres repeticiones. El mejor tratamiento utilizado fue la estrategia combinada siendo la más eficiente y la que logró mejores resultados en el rendimiento llegando a obtener un promedio de 6.4-9.5 t/ha. La altura de planta en el T2 y T1 estuvo entre los 51.7 y $51.4 \mathrm{~cm}$ respectivamente. La estrategia combinada controló efectivamente la incidencia del tizón en las tres localidades con un porcentaje de 20-29\%, en comparación con el T1 y T0 que tuvo el $25 \%$ y $40 \%$, respectivamente. Se debe tomar en cuenta que el uso de bioinsumos contribuye a la producción limpia y sostenibilidad del sistema.

Palabras clave adicionales: Estrategia, producción, ecológico, cosecha, bioinsumo.

\section{Ecological management strategies for late blight potato [Phytophthora infestans (Mont.) De Bary]}

\section{Summary}

In order to evaluate ecological strategies of Late Blight control for improving production and profitability of potato crop (Solanum tuberosum var. Huaycha), in three localities of the Municipality of Independencia-Ayopaya-Cochabamba, soil preparation was performed, as well as plot layout, planting, fertilization, cultural practices (irrigation, weed control, weeding, phytosanitary control where treatments we applied) and harvest for further

\footnotetext{
Autor para correspondencia. Correo electrónico: n.ortuno@umss.edu.bo Estudiante FCAP-UMSS

Investigadores Fundación PROINPA, Cochabamba, Bolivia.

Docente-investigador FCAP-UMSS, Cochabamba, Bolivia.
} 
evaluation. Three treatments (T0 witness, T1 organic strategy and T2 combined strategy) in a design of randomized complete blocks with three replications were evaluated. The best treatment used was the combined strategy being the most efficient and that achieved better results in performance reaching an average of 6.4-9.5 tn / ha. The plant height in T2 and T1 was between 51.7 and $51.4 \mathrm{~cm}$ respectively. The combined strategy effectively controlled the incidence of late blight in the three locations with a percentage of 20 to $29 \%$, compared to the T1 and T0 which had $25 \%$ and $40 \%$ respectively It should be taken into account that the use of bio-inputs contributes to the clean production and sustainability of the system.

Additional Key words: Estrategia, producción, ecológico, cosecha, bioinsumo.

\section{Introducción}

En Bolivia, el cultivo de papa es muy importante desde el punto de vista histórico, social, económico y alimenticio. Actualmente la producción de la papa involucra a mas de 200.000 familias que cultivan un total de 129.230 ha. En siete de los departamentos del país, la producción anual de la papa es de 827.690 t (Crespo et al., 2005). A nivel nacional, el departamento de Cochabamba está considerado como el principal productor de papa, tanto para semilla como también para el consumo. La superficie promedio cultivada en el departamento alcanza aproximadamente a 21624 ha, con un rendimiento promedio de 5,01t/ha, el cual es considerado uno de los más bajos a nivel nacional (Toromayo, 2006 y Gabriel et al., 2011 b).

Las estadísticas citadas demuestran que la papa constituye uno de los alimentos más importantes de la población urbana y rural de Bolivia, pero la producción es baja debido a factores diversos, entre los cuales se encuentran los abióticos y bióticos y el alto costo de los insumos externos, como son los plaguicidas y fertilizantes (Bolleta et al., 2005). Benzing (2001), indica que el 33,8\% de los productores agropecuarios se dedican a la producción de la papa. El grupo de agricultores de subsistencia, localizados mayormente en la sierra media y alta, cuyos costos de producción son mínimos, tienen bajos rendimientos (3 a 6 t/ha), debido a problemas de erosión del suelo, condiciones agroclimáticas adversas (sequía y heladas) y a la presencia de plagas y enfermedades (Gabriel et al., 2011a). La producción de papa en el Departamento de Cochabamba, se ha visto amenazada por la presencia del Tizón (Phytophthora infestans) y de las plagas como el Trips y los Epitrix que disminuyen el rendimiento significativamente, lo cual obliga al productor a aplicar pesticidas para el control de plagas (Toromayo, 2006 y Gabriel et al., 2011 a).

Para lograr una producción aceptable el productor recurre al uso intensivo e inadecuado de agroquímicos sintéticos de diferente índole, provocando varios problemas que afectan negativamente a su calidad de vida (Gabriel et al., 2011 b). Entre estos problemas podemos citar el incremento considerable de los costos de producción, debido al elevado precio de los agroquímicos que son generalmente importados, otro problema es la intoxicación accidental de los agricultores por el uso de agroquímicos que son utilizados sin las precauciones y cuidados correspondientes. A ello además debemos sumarle los efectos de la contaminación ambiental que provocan la degradación biológica del suelo y el agua (Bolleta et al., 2005). 
Ante esta situación es cada vez mayor y urgente la creación de nuevas alternativas tecnológicas que sean más amigables con el ambiente, menos costosas y que permitan mantener y/o mejorar los rendimientos (Bolleta et al., 2005). La necesidad de disminuir la dependencia del uso de productos químicos artificiales en los distintos cultivos está obligando a buscar alternativas de solución como el uso de bioinsumos para el control de plagas y enfermedades, que permiten aprovechar elementos naturales que al ser elaborados brindan mayor confianza al productor y son eficientes para el debido control de enfermedades como la Phytopthora infestans, que es un problema generalizado en el país en el cultivo de papa, es llamado también tizón, T'octu, pasmo negro, qhasu, mal negro (Toromayo, 2006).

Existen algunos antecedentes que demuestran que es posible reducir el uso de agroquímicos en el cultivo de la papa cambiando insumos orgánicos con químicos para el control del tizón y mejorar la productividad de la papa (Bolleta et al., 2005, Mamani et al., 2016). Dar un ejemplo específico. Asimismo, varias investigaciones brindan evidencias de que reactivando la biología del suelo y mejorando la nutrición de las plantas, se fortalecen los tejidos y ello mejora la capacidad de las plantas para soportar el ataque de enfermedades y plagas. En resumen se requiere un manejo mas integral del cultivo para reducir el uso de agroquímicos alcanzando niveles productivos aceptables.

\section{Materiales y métodos}

El presente trabajo de investigación se realizó en tres comunidades (Huancarani, Link'u y Machaca) del Municipio de Independencia, primera sección de la Provincia Ayopaya, ubicado a $245 \mathrm{Km}$. de la capital del departamento de Cochabamba, donde la temperatura media es de 16 a $18^{\circ} \mathrm{C}$, la precipitación pluvial media anual es de $600 \mathrm{~mm}$, la altitud oscila entre 3.600 y $4.250 \mathrm{msnm}$; la tenencia de tierra es de 1 a 2 hectáreas por familia y la superficie cultivada es de aproximadamente 3337 hectáreas (Consorcio Interinstitucional, 2008).

Estas zonas se caracterizan por tener un clima variado (templado, lluvioso a seco) los ensayos presentan un clima templado a frígido de 2400 a 3800 m.s.n.m. (carta $\mathrm{n}^{\circ} 6242$ del IGM), la precipitación promedio anual alcanza a $841,75 \mathrm{~mm}$, en los meses de noviembre a marzo (Rojas, 1995). Los suelos son generalmente franco arenosos y en algunos lugares arcillosos. La sub Central Huancarani, presenta zonas erosionadas, los cuales son principalmente de origen hídrico de tipo laminar, y en surcos lo que ocasionan la formación de cárcavas y en menor grado deslizamientos. Las causas principales son el sobre pastoreo de laderas erosionadas y la pérdida de vegetación (Consorcio Interinstitucional, 2008).

Para la realización del presente trabajo de investigación, se utilizó semilla de papa variedad Huaycha, Categoría Registrada 2, procedente de la asociación de productores SEPA y los insumos Vigortop (biofertilizante foliar), Fungitop (biofungicida orgánico) y Ridomil (fungicida sistémico).

El diseño experimental implementado fue el de bloques completos al azar en tres localidades (Huancarani, Link'u y Machaca), con tres tratamientos (estrategias de control). Cada unidad experimental estuvo constituida por 10 surcos de $7 \mathrm{~m}$ de largo, $3 \mathrm{~m}$ de ancho, siendo la distancia entre surcos $0.7 \mathrm{~m}$, la distancia entre plantas $0.3 \mathrm{~m}$ y el área de $21 \mathrm{~m}^{2}$ por unidad experimental. La unidad 
de muestreo fue de 10 plantas al azar dentro de cada tratamiento y en cada localidad.

Para la preparación del suelo en las tres localidades se utilizó el sistema tradicional, es decir yunta de bueyes, con arado de palo; realizando tres pasadas una semana antes de la siembra. Una vez nivelado el terreno, se procedió al dimensionamiento de los bloques y las unidades experimentales. Se surcó con yunta y arado de palo, posterior a ello se procedió a la siembra, derramando la semilla a una distancia de $0.30 \mathrm{~m}$ entre semilla y semilla, aplicando estiércol ovino (5 t/ha) de uso local a todos los bloques y en las tres localidades en las que se instaló los cultivos, brindándoles de esta manera las mismas condiciones para su respectiva comparación.

La fertilización se la realizó con Vigortop (5 L/ha), en un promedio de 5 aplicaciones efectuadas después de la emergencia, cada 10 días (Ortuño et al., 2011). El Fungitop fue aplicado como fungicidad de contacto (5 L/ha) (Ortuño et al., 2008) y el Ridomil, fungicida sistémico (dosis comercial), siguiendo las recomendaciones de Fernández-Northcote et al. (1999). El riego fue proporcionado según la necesidad del cultivo con una frecuencia de tres días. El tipo de riego aplicado fue por aspersión en cada localidad. El control de malezas fue mediante carpidas y deshierbes con herramientas manuales cuando las plantas alcanzaron una altura de 2 a 3 centímetros. Se realizó un solo aporque, por costumbre local, en forma manual a los 60 días después de la siembra (DDS), cuando las plantas alcanzaron una altura de $15-25 \mathrm{~cm}$, antes de inicio de la tuberización para favorecer la formación del tubérculo.

Los tratamientos estuvieron constituidos por las siguientes estrategias de control de Tizón:

T $0 \rightarrow$ Testigo (sin ninguna aplicación); T $1 \rightarrow$ Estrategia de control orgánica o ecológica; basada en la aplicación de Vigortop (biofertilizante - promotor de crecimiento) y Fungitop (biofungicida orgánico); T $\mathbf{2} \rightarrow$ Estrategia de control combinada; basada en Vigortop (biofertilizante - promotor de crecimiento), Fungitop (biofungicida orgánico) y Rhidomil (fungicida sintético sistémico). La aplicación de fungicidas (químicos y orgánicos) se efectuó a los 40 días luego de en dos aplicaciones (40 y 52 días), preventivas, usando una mochila manual de 20 litros de capacidad (Tabla $1)$.

Tabla 1. Cronograma de aplicación de fertilizantes orgánico y fungicidas para el control de Tizón en cultivo de la papa.

\begin{tabular}{llll}
\hline FECHA & T0 & T1 & T2 \\
& TESTIGO & $\begin{array}{l}\text { CONTROL } \\
\text { ORGANICO }\end{array}$ & $\begin{array}{l}\text { CONTROL } \\
\text { COMBINADO }\end{array}$ \\
\hline 1.- 10 días DDE & S/C & Vigortop & Vigortop \\
2 .- 20 días DDE & S/C & Vigortop & Vigortop \\
$\mathbf{3}$.- 30 días DDE & S/C & Vigortop & Vigortop \\
$\mathbf{4 . -}$ 40 días DDE & S/C & Vigortop + Fungitop & Vigortop + Fungitop + \\
& & Fungitop & Fungitop + Rhidomil \\
5.- 52 días DDE & S/C & Funil & \\
\hline
\end{tabular}


La cosecha se realizó, extrayendo los tubérculos manualmente con la "Chujchuka", al final del ciclo productivo en cada localidad cosechándose solo los 3 surcos centrales para evitar el efecto de bordura.

En base al modelo estadístico mencionado, se realizó el análisis de varianza (ANVA) para cada variable de respuesta y se probó la significancia de los efectos fijos. La estimación de los componentes de varianza se realizó mediante el método restringido de maximización de la función de probabilidad, previa verificación de los supuestos de normalidad y homogeneidad de varianzas, utilizando el PROC glm de SAS (SAS, 2004).

De acuerdo con los objetivos de la investigación las variables de respuesta evaluadas fueron:

Altura de planta, se evaluó en la unidad de muestreo, midiendo desde la base hasta el ápice de la planta utilizando un flexómetro cada 15 días después de la emergencia, hasta el inicio de la floración. Porcentaje de cobertura foliar, se evalúo en cada unidad experimental a medida que incrementaba el desarrollo del follaje, a la misma frecuencia de altura de la planta, utilizando un bastidor cuadrangular de madera diseñado de $1 \mathrm{~m} x$ $1 \mathrm{~m}$ por lado, tomando en cuenta el porcentaje de follaje existente en el metro cuadrado evaluado. Porcentaje de incidencia del tizón, se evaluó cada 10 días, por cinco veces, observando visualmente en la unidad de muestreo, para ver qué cantidad de plantas fueron afectadas por la presencia de una condición, síntoma de enfermedad o alguna lesión provocada por el Tizón. El porcentaje de severidad y de incidencia se evaluaron al mismo tiempo en cada unidad de muestreo, Utilizando las siguientes formulas (Gabriel et al., 2011a).

\section{$\%$ de severidad $=\underline{\text { Número de hojas enfermas } * 100}$ \\ Superficie total de la planta \\ $\%$ de incidencia $=\underline{\text { Número de plantas enfermas por parcela* } 100}$ \\ Número total de plantas observadas por parcela}

Para la evaluación del rendimiento se consideró los seis surcos centrales de cada unidad experimental; cosechándose según las categorías mencionadas por el agricultor y mercado regional de Cochabamba (Chapara, Kolqué, Murmu, Ch'ilimurmu y Ch'ili), cuantificando el número de tubérculos $\mathrm{y}$ peso de tubérculos por tamaño, con el fin de establecer la relación de productividad con el uso de biofertilizantes orgánicos. Además se descartó las papas dañadas al realizar la cosecha en la unidad experimental.

\section{Resultados y discusión}

A continuación, solo se presentan los resultados que fueron estadísticamente significativos. En la altura de planta solo se analizó la altura planta antes de la floración, donde se observaron diferencias altamente significativas $(\mathrm{p}=0.01)$ en la interacción entre localidades $\mathrm{x}$ estrategias. La figura 1, muestra mayor altura de plantas en la localidad de Machaca, seguida por Huancarani en la cual tuvo mejores condiciones climáticas que en Link'u. Una mayor altura de planta en la 
estrategia combinada en las localidades de Machaca y Huancarani por contar estas con mejores condiciones climáticas. Entre localidades la comparación de la altura entre las estrategias de control, con un 99\% de seguridad se pudo evidenciar que existen diferencias altamente significativas entre localidades y las estrategias de control, indicando de esta manera que los tratamientos tuvieron diferencias leves entre el tratamiento orgánico y el combinado a diferencia del testigo en el cual el promedio de la altura fue menor que los otros 2 tratamientos. Las diferencias existentes entre tratamientos hacen suponer que su causa está muy ligada a la aplicación del biofertilizante (Vigortop), aplicado en 2 de los tratamientos en los cuales se favoreció el desarrollo de las plantas

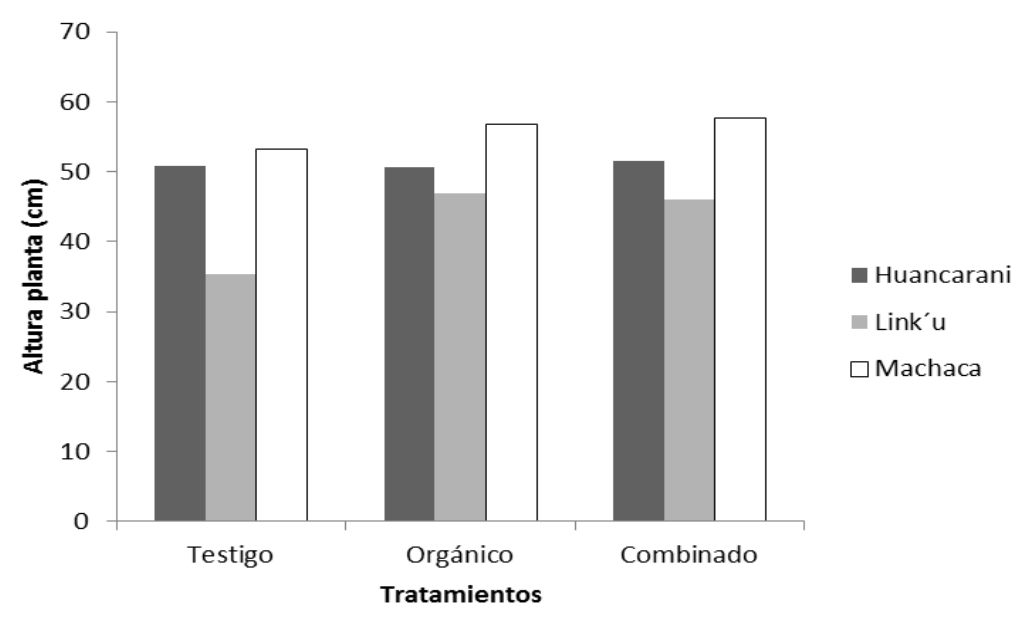

Figura 1. Altura de planta entre localidades y estrategias de control.

Cuando se analizó la altura de planta, entre tratamientos, también se encontró diferencias significativas $(\mathrm{p}=0.05)$ entre las estrategias de control de Tizón, teniendo un mejor resultado con la aplicación de los tratamientos combinado y orgánico, respecto al testigo, como se demuestra en la figura 2 


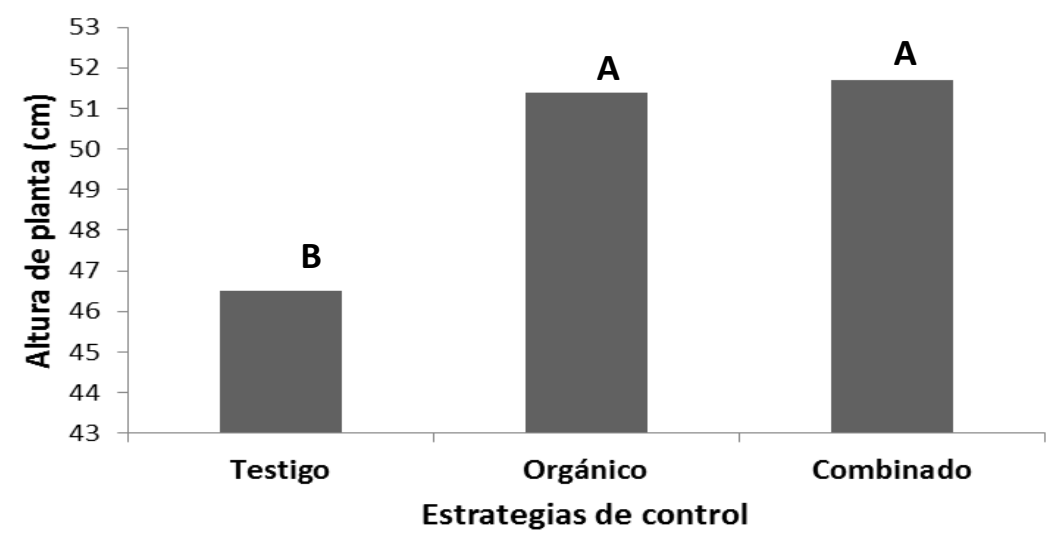

Figura 2. Efecto de estrategias de control sobre la altura de planta.

Wiersema y Cabello (1985), establecieron que existe una alta correlación entre el número de tallos y la producción de tubérculos. Según los datos mostrados únicamente se observó diferencia altamente significativa para la variable altura de planta a los 25 días después de la siembra, con $46.5 \mathrm{~cm}$ para plantas correspondientes al testigo, $51.4 \mathrm{~cm}$ para las plantas de la estrategia orgánica y con $51.7 \mathrm{~cm}$ para las plantas de la estrategia combinada. Este resultado probablemente obedezca al hecho de que el biofertilizante, Vigortop, utilizado por su mayor grado de solubilidad, estaba disponible en cantidades mayores, en la etapa temprana del desarrollo del cultivo.

En la cobertura foliar, fueron analizados los datos antes de la floración, los que presentaron diferencias significativas entre localidades y entre estrategias $(\mathrm{p}=0.05)$, y no así en la interacción localidad $\mathrm{x}$ estrategia.

Se observó un mayor porcentaje de cobertura foliar en la localidad de Machaca, posiblemente por las condiciones del suelo, en Link'u se tuvo un $34 \%$ de cobertura, aunque se debe mencionar que después de la lectura de datos hubo una granizada que afecto al rendimiento en esta localidad (figura 3 ).

En la fase del crecimiento vegetativo del cultivo, se refleja la eficiencia con la que se nutre la planta y el efecto de los factores ambientales durante el ciclo del cultivo, siendo el incremento en tamaño y volumen de la planta de papa en producto de la acumulación de biomasa (Theodoracopoulos et al., 2008). 


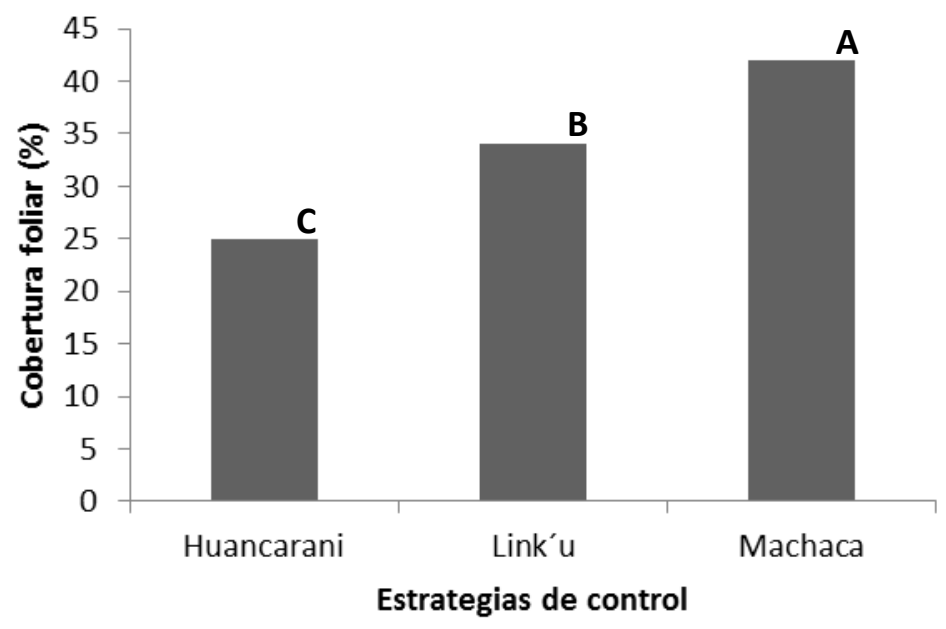

Figura 3. Porcentaje de cobertura foliar entre las localidades.

Hubo diferencias significativas entre con respecto a las otras estrategias de estrategias $(\mathrm{p}=0.05)$, existiendo mayor control de Tizón (figura 4). cobertura foliar en la estrategia orgánica

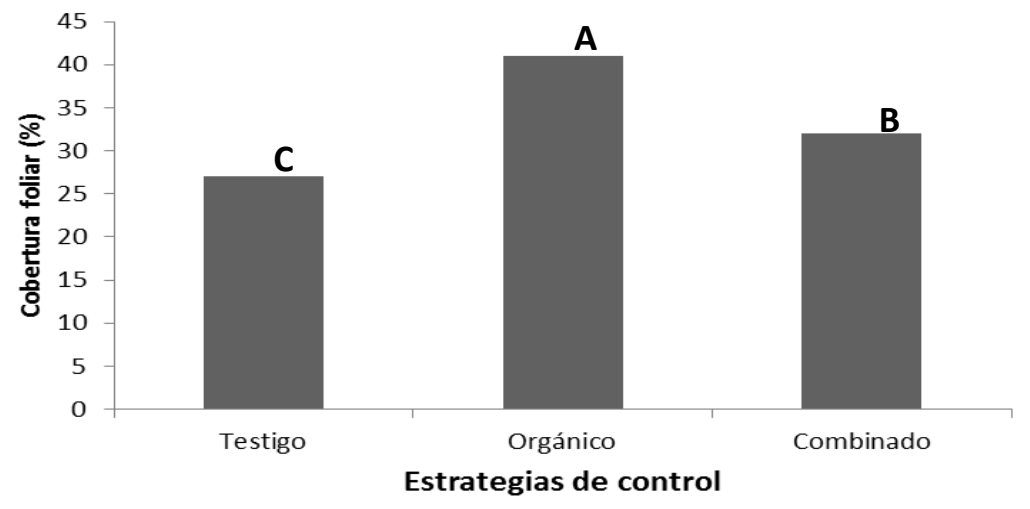

Figura 4. Porcentaje de cobertura foliar en relación en las estrategias de control.

En la incidencia de la enfermedad, no se observaron diferencias significativas en la interacción localidades x estrategias, pero sí entre localidades, se observaron diferencias estadísticas $(\mathrm{p}=0.05)$. En la figura 5 , se observa la incidencia del tizón en el Testigo, en las tres localidades, siendo la menor en la estrategia combinada. Gabriel et al. (2011a), menciona que la incidencia es importante, desde el punto de vista comparativo en pruebas de control de enfermedades, mas entre localidades. 


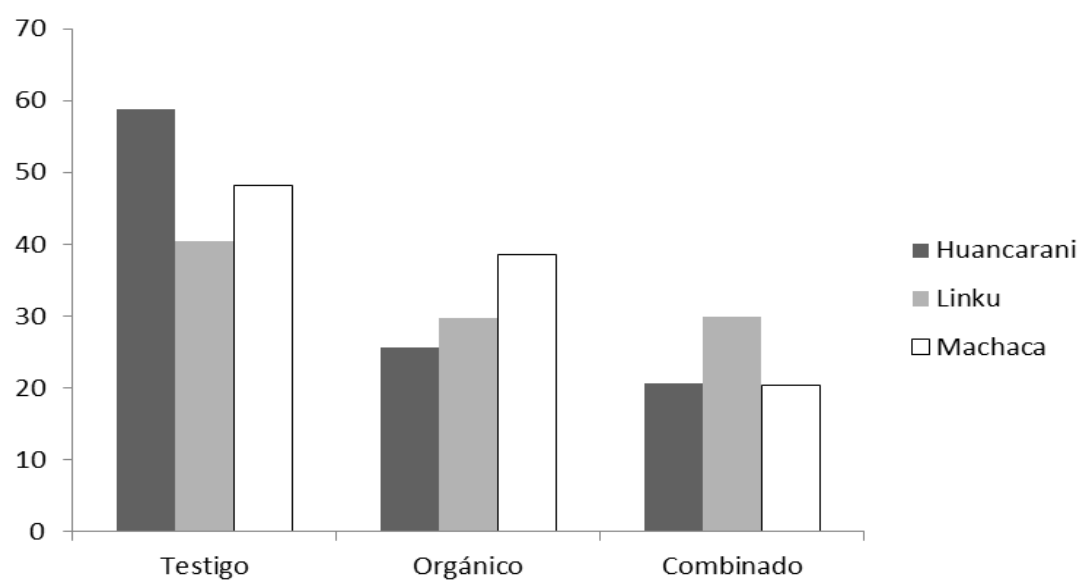

Figura 5. Incidencia de la enfermedad entre localidades y estrategias de control de tizón tardío en cultivo de la papa.

En la incidencia de la enfermedad, existieron diferencias significativas $(p=0.05)$ entre estrategias. En la figura 6, se muestra que hubo mayor incidencia del tizón en el Testigo (sin aplicación) con un $48 \%$; en cambio hubo menor incidencia en la estrategia orgánica seguida por la combinada.

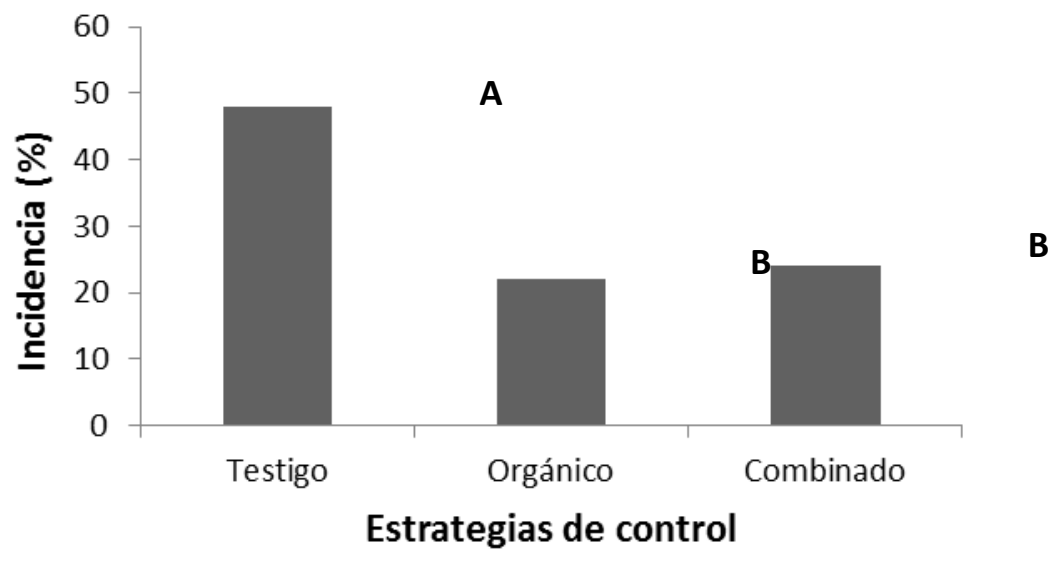

Figura 6. Porcentaje de incidencia de tizón tardío en el cultivo de papa por estrategia utilizada.

En el porcentaje de severidad del tizón, no existieron diferencias significativas entre localidades $\mathrm{x}$ estrategias, ni entre localidades. Sin emabrgo, observando entre estrategias se observaron diferencias $(\mathrm{p}=0.05)$, donde el Testigo fue mayor, seguido por la estrategia orgánica en los cuales el porcentaje fue aumentando a medida que transcurrían los días a diferencia de la estrategia combinada en la que el porcentaje fue menor porque el control fue más efectivo y se mantuvo casi constante (figura 7). 


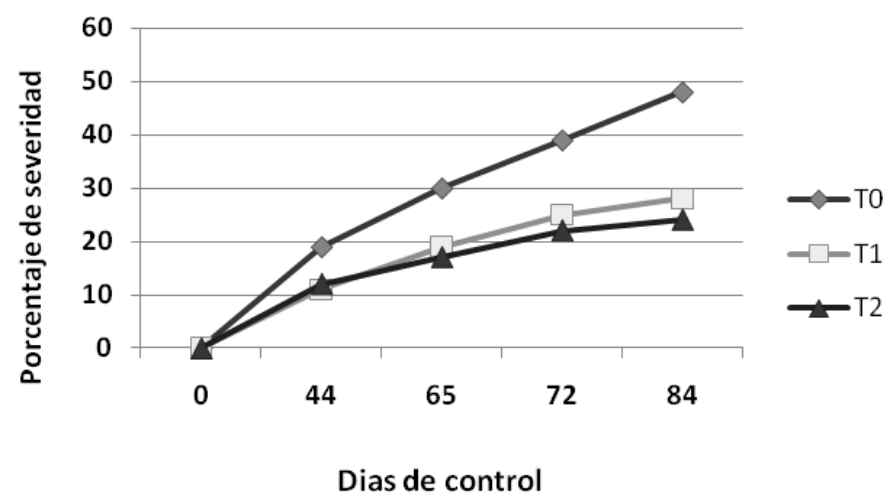

Figura 7. Efecto de estrategias sobre el porcentaje de severidad del tizón tardío en el cultivo de papa.

Los primeros síntomas visibles del tizón se observaron a los 44 días después de la siembra, incrementándose en forma gradual hasta los 65 días, con presencia en todos los tratamientos a partir de los 72 días. En el tratamiento T-0, se observó un incremento acelerado, alcanzando un nivel máximo de $48 \%$ de daño a los 84 días. En cambio, en los tratamientos T-1 y T-2 fueron moderados.

En el rendimiento del cultivo de papa, según el análisis de varianza, hay diferencias significativas $(p=0.05)$ en el rendimiento entre localidades $\mathrm{x}$ estrategia. La figura 6, se muestra un mayor rendimiento con la estrategia combinada en las 3 localidades, siendo el rendimiento mayor en la localidad de
Huancarani, debido a que la combinación del Fungitop (biofungicida de contacto), con el Ridomil (fungicida sintético sistémico), mejoró considerablemente los rendimientos, pero estos rendimientos variaron en función al número de aplicaciones de los fungicidas. El número de aplicaciones de las estrategias fueron 2, lo cual parece insuficiente, pues realizando mas aplicaciones el ataque de Tizón se hubiera reducido y el rendimiento hubiera incrementado por que se perdieron muchas plantas, especialmente en la localidad de Link'u.

La estrategia de control combinado fue la mejor en las tres localidades, seguida por la estrategia orgánica, teniendo un mejor rendimiento en Huancarani. 


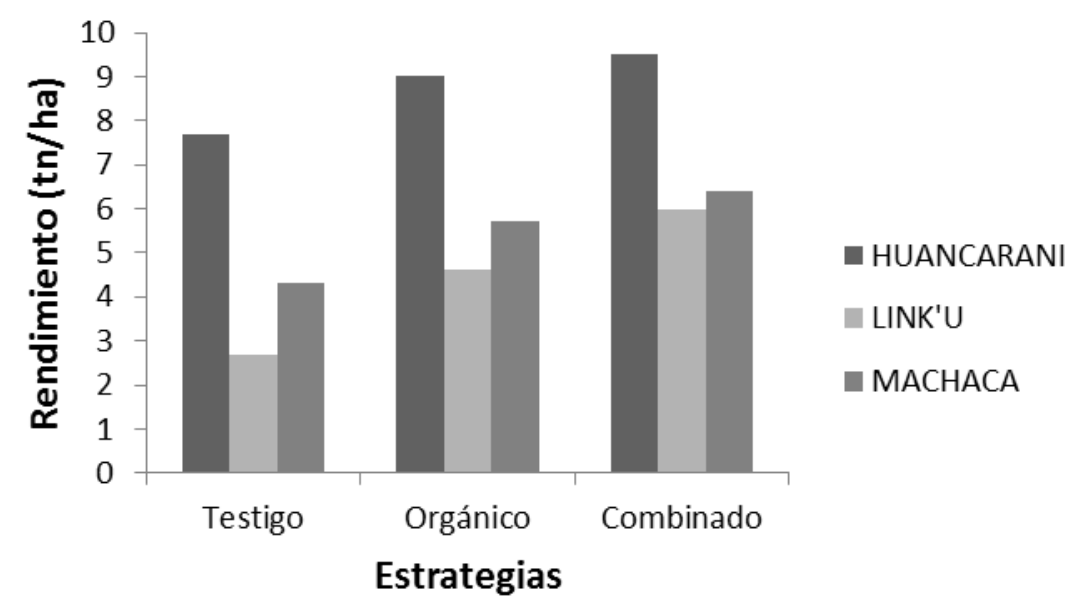

Figura 6. Efecto de las estrategias de control y las localidades sobre el rendimiento en el cultivo de papa.

Según la figura 7, en Huancarani hubo mayores rendimientos en las categorías "Chapara", "Kolque" por existir mejores condiciones climáticas, siendo las mejores las estrategias combinada y orgánica.

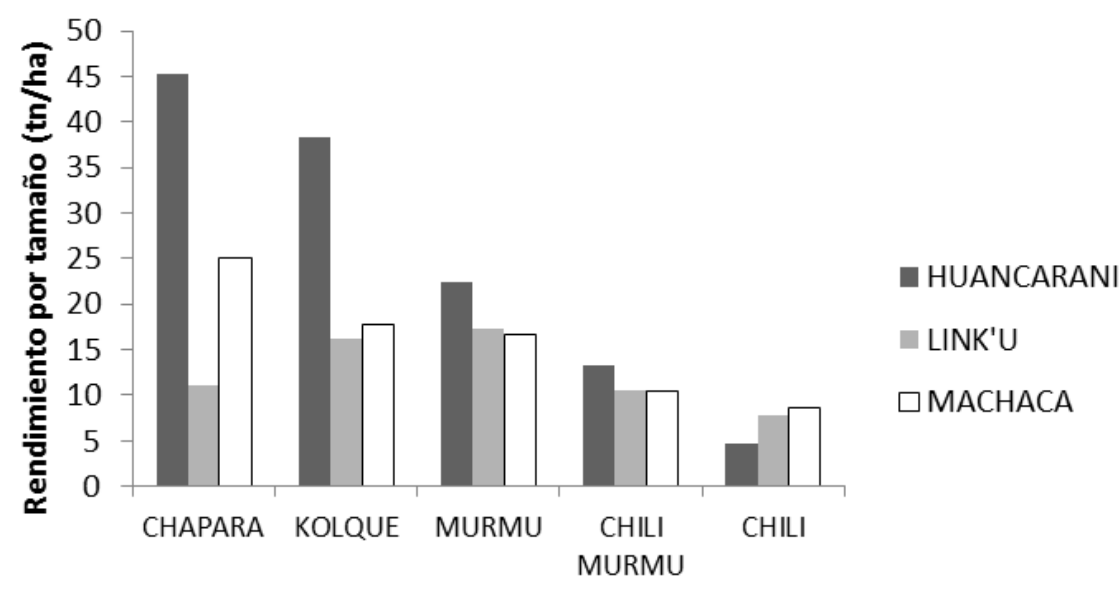

Tamaño de tubérculos a la cosecha

Figura 7. Rendimiento por categoría respecto a las estrategias de control.

\section{Conclusiones}

De acuerdo a los resultados obtenidos tanto de la evaluación técnica y económica, se tiene las siguientes conclusiones:
La estrategia de control de tizón combinada (orgánico y sintético), fue la más eficiente y la que logró mejores resultados en el rendimiento llegando a obtener entre 6.4 a $9.5 \mathrm{t} / \mathrm{ha}$. 
En las variables altura de planta, la estrategia combinada y orgánica alcanzaron alturas de 51.7 y $51.4 \mathrm{~cm}$ respectivamente y el testigo llego solo a $46 \mathrm{~cm}$. Sin embargo la cobertura foliar no tuvo diferencias significativas entre tratamientos.

El tratamiento combinado (orgánicosintético) logró controlar efectivamente la incidencia del tizón en las tres localidades, llegando a tener un porcentaje de incidencia de solo 20-29\%, en comparación con el tratamiento orgánico (25-38\%) y por último el testigo tuvo un mayor porcentaje de incidencia con $40-53 \%$.

La promoción de los bioinsumos no sólo contribuye a incrementar el ingreso de los productores y la calidad de vida, sino que con ello también se da cabida a una mayor competitividad de todo el sector agropecuario en el mercado internacional, al implementar sistemas productivos limpios y con la posibilidad que ellos puedan producir sus propios biofungicidas.

El costo del tratamiento combinado es mayor que el tratamiento orgánico con $280 \mathrm{Bs}$, mientras que el beneficio neto entre el T2 y el T1 varia con 1792 Bs.

\section{Conflicto de intereses}

No se tiene ningún conflicto de intereses con ningún sector.

\section{Referencias citadas}

Aguilar, L. E. 2005. Uso de caldos minerales y extractos naturales para el control del tizón tardío (Phytophthora infestans); de la papa en Colomi-Bolivia. Tesis Ing. Agro. Universidad Mayor de San Simón. Facultad de Ciencias Agrícolas y Pecuarias. CochabambaBolivia. Pág. 1-55.
Benzing, A. 2001. Agricultura orgánica: Fundamentos para la región andina. Editorial Neckar - Verlag, Villingen Schewenningen - Alemania. 628 p.

Bijanic, A. 2005. Sondeo sobre demanda nacional de semilla de papa para el sector formal y su pertinencia para la UPSSEPA. Informe: Cooperación Técnica Suiza. La Paz, Bolivia.

Bolleta, A.; Krüger H.; Venanzis, S. 2005. Respuestas de un cultivo de avena en siembra directa a la fertilización química y biológica en un ambiente marginal. Disponible en: http://www.inta.gov.ar (accesado el 20 de diciembre 2010).

Carrasco, E.; Estrada, N.; Gabriel, J.; Larondelle, Y.; García, W.; Quiroga, O. 1997. Seis cultivares potenciales de papa con resistencia al Tizón (Phytophthora infestans) en Bolivia. Revista Latinoamericana de la Papa 9/10: 106122.

Consorcio Interinsticional (AGRUCO, PROINPA, BASFOR/ESFOR). 2008. Diagnóstico participativo de las sub centrales Huancarani y Machaca, Municipio de Independencia. Proyecto Biocultura "Proinpa". Cochabamba-Bolivia. 133p.

Crespo, F. y Bellot, C. 2005. La agricultura sostén de la economía. Disponible en: http://www.nuevaeconomia.com.bo/docs/ 05agrop.pdf. (accesado el 20 de diciembre 2009).

ICA - Instituto Colombiano Agropecuario. 2004. Control de los Bioinsumos y Extractos Vegetales de uso agrícola en Colombia. Bogotá. Disponible en: www.ica.gov.co. (Actualización diaria, accesado el 24 de marzo 2011).

INTA, 2002. Informe Técnico Anual 1999-2000. Guía Tecnológica de cultivo 
de la papa. Estelí, Nicaragua. Disponible en: http://www.inta.gov.ar (accesado el 20 de diciembre 2010).

Fernandez-Northcotte, E. y Navia, O. 1995. Manejo Integrado de Plagas: Manejo Integrado del Tizón. En: Memorias de II Curso de Manejo Integrado de Plagas de Papa. Curso de Capacitación a Distancia. 1995. IBTA PROINPA. Cochabamba, Bolivia. 165 p.

Gabriel, J.; Fernandez, S,; Plata, G.; Siles, M. 2011a. Niveles de resistencia al Tizón tardío en clones de papa del Centro Internacional de la Papa evaluados en Bolivia. Revista Latinoamericana de la Papa 16(1): 126-141.

Gabriel, J.; Orellana, L.; Plata, G.; Siles, M. 2011b. Aptitud combinatoria de resistencia a tizón tardío (Phitophthora infestans) en cultivares nativos de papa. Revista Latinoamericana de la Papa 16(1): 85-98.

Medrano, A.; Ortuño, N. 2007. Control de Damping Off, mediante la aplicación de bioinsumos en almácigos de cebolla en el valle Alto de Cochabamba-Bolivia. Revista de Ciencias y Tecnología ACTA NOVA. Universidad Católica Boliviana San Pablo. Vol.3, N ${ }^{\circ} 4$.

Nina, H.F. 2004. Rendimiento de papa con el empleo de abonos orgánicos y fertilizantes mineral en localidad de Huankuri cantón Challa Grande (Provincia Tapacari). Tesis Ing. Agr. Universidad Mayor de San Simón. Facultad de Ciencias Agrícolas y Pecuarias. Cochabamba-Bolivia. Pág. 135.

Organo oficial de la Asociación Latinoamericana De Fitopatologia- ALF. 2006. Integración de nuevas estrategias de manejo del Tizón de la papa (Phytophthora infestans) y del suelo para una agricultura sostenible Vol. 41, No 3 .
Ortuño, N.; Navia, N.; Medrano, A.; Rojas, K.; Torrico L. 2008. Desarrollo de bioinsumos: Un aporte a la soberanía alimentaria de Bolivia. Revista de Agricultura. Año 62, No 47.

Ortuño, N.; Navia, O.; Meneses, E.; Barja, D.; Villca, S.; Plata, G.; Claros, M.; Gutierrez, C.; Arandia, W.; Crespo, L. 2011. Catálogo de bioinsumos. Cochabamba, Bolivia. 42p.

Mamani, E.; Morales,V.; Ortuño, N. 2016. Aplicación de biofertilizantes foliares en el cultivar Huanch'a (Solanum tuberosum andigena) en los valles interandinos de Bolivia. Revista Latinoamericana de la papa. 21(2):14-25.

Ramírez, L. y Soto, G. 2000. Una experiencia de papa orgánica en la zona de Cartago, Costa Rica. En: www.infoagro.com/hortalizas. (Accesado el 18 de abril 2011).

Restrepo, J. 2000. Agricultura orgánica una Teoría y una Práctica. Cali, Colombia. Pág. 56, 127, 128.

Rivera, R.; Fernandez, E; Hernandez, A.; Martin, J.R. 2003. El manejo eficiente de la simbiosis Micorriza, una vía hacia la agricultura sostenible estudio de caso: El Caribe. Ediciones INCA, La habana Cuba. 189 p.

SAS Institute Inc. SAS/STAT. 2004. User Guide, versión 9.1, Four Edition, Vol 2, SAS Institute Inc., Cary, N.C. 2004.

Theodoracopoulos, M.; Arias, S.; Avila, H. 2008. Manual de producción de papa. Disponible en: www.hondurasag.org y www.fintrac.com. (Accesado el 18 de abril 2011).

Toromayo, T.E. 2006. Evaluación agronómica de siete variedades de papa Solanum tuberosum en dos localidades de la provincia Bolívar. Tesis Ing. Agr. 
Universidad Mayor de San Simón. Pecuarias. Cochabamba - Bolivia. Pág 1Facultad de Ciencias Agrícolas y 25. 